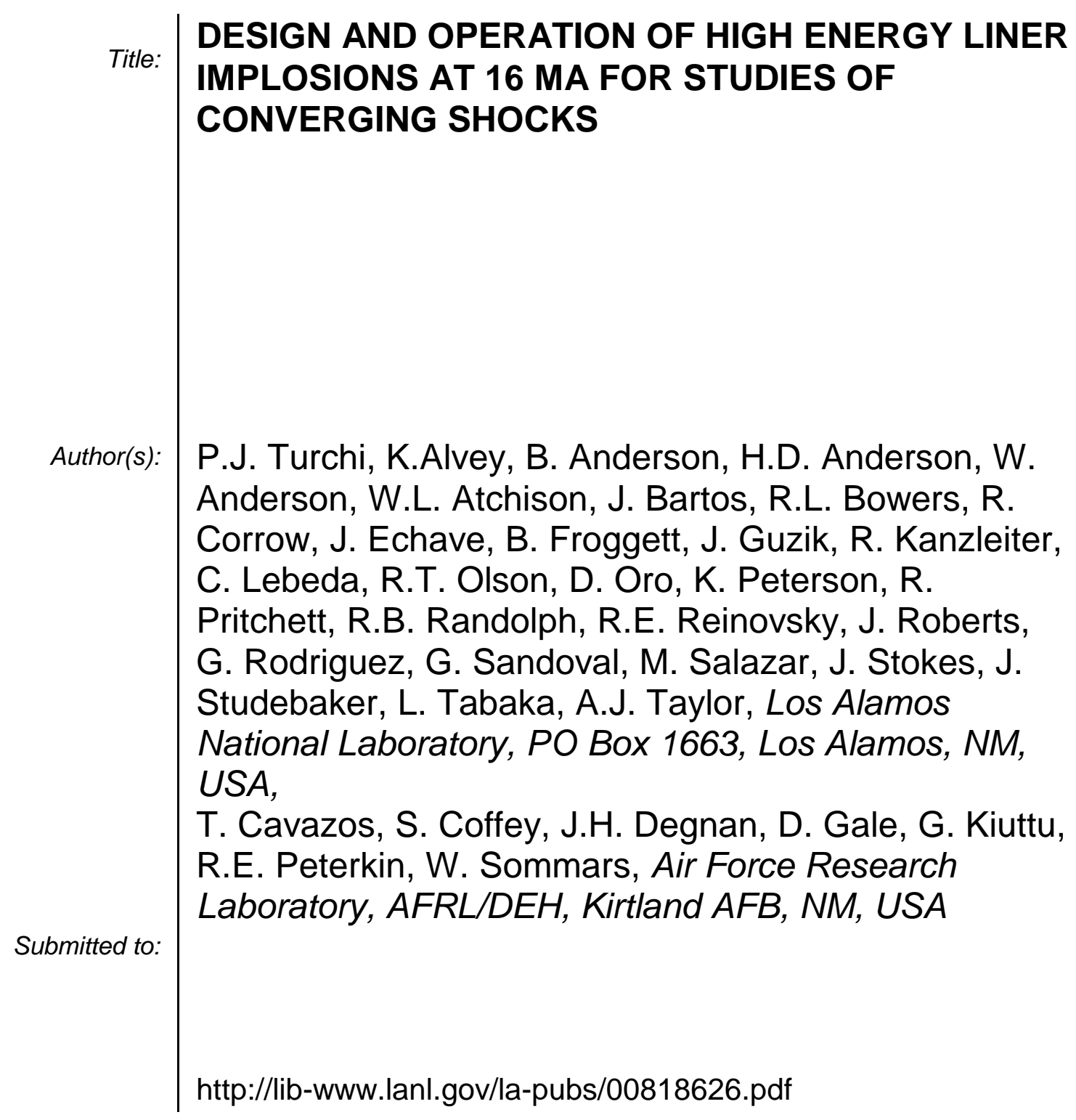

Los Alamos National Laboratory, an affirmative action/equal opportunity employer, is operated by the University of California for the U.S. Department of Energy under contract W-7405-ENG-36. By acceptance of this article, the publisher recognizes that the U.S. Government retains a nonexclusive, royaltyfree license to publish or reproduce the published form of this contribution, or to allow others to do so, for U.S. Government purposes. Los Alamos National Laboratory requests that the publisher identify this article as work performed under the auspices of the U.S. Department of Energy. Los Alamos National Laboratory strongly supports academic freedom and a researcher's right to publish; as an institution, however, the Laboratory does not endorse the viewpoint of a publication or guarantee its technical correctness. 


\title{
DESIGN AND OPERATION OF HIGH ENERGY LINER IMPLOSIONS AT 16 MA FOR STUDIES OF CONVERGING SHOCKS*
}

\author{
P.J. Turchi, K.Alvey ${ }^{1}$, B. Anderson, H.D. Anderson², W. Anderson, W.L. Atchison, J. Bartos, \\ R.L. Bowers, R. Corrow ${ }^{1}$, J. Echave ${ }^{2}$, B. Froggett ${ }^{2}$, J. Guzik, R. Kanzleiter, C. Lebeda, \\ R.T. Olson, D. Oro, K. Peterson ${ }^{1}$, R. Pritchett ${ }^{1}$, R.B. Randolph, R.E. Reinovsky, J. Roberts, \\ G. Rodriguez, G. Sandoval, M. Salazar, J. Stokes, J. Studebaker, L. Tabaka, A.J. Taylor, \\ Los Alamos National Laboratory, PO Box 1663, Los Alamos, NM, USA, \\ T. Cavazos $^{3}$, S. Coffey ${ }^{4}$, J.H. Degnan, D. Gale ${ }^{3}$, G. Kiuttu, R.E. Peterkin, W. Sommars ${ }^{3}$, \\ Air Force Research Laboratory, AFRL/DEH, Kirtland AFB, NM, USA
}

Abstract

Electromagnetically-driven implosion of soliddensity, cylindrical liners can launch shocks with excellent precision at impact speeds exceeding $5 \mathrm{~km} / \mathrm{s}$. We discuss the design and operation of liner implosions driven at peak currents of 16MA, using the Shiva Star capacitor bank at the Air Force Research Laboratory. Liners of 1100 aluminum, with initial length, radius and thickness of $4 \mathrm{~cm}, 5 \mathrm{~cm}$ and $1 \mathrm{~mm}$, respectively, implode under the action of an axial current, rising in $8 \mu \mathrm{s}$. Fields on conductor surfaces exceed 0.6 MG. The inner surface of the liner achieves a speed of $6.25 \mathrm{~km} / \mathrm{s}$ when it impacts a concentric target cylinder of tin at a radius of 2 $\mathrm{cm}$. Magnetic probes and radially-aligned X-radiography follow the motion of the liner and its impact on the tin cylinder. This cylinder holds a solid cylinder of acrylic of $1.5 \mathrm{~cm}$ radius in which the motion of a converging shock is followed by optical shadowgraphy and axiallyaligned, X-radiography.

Design issues that were successfully addressed include: Pulsed Power - current joints at high magnetic fields in the vicinity of the liner and glideplane/electrodes, where magnetic pressures quickly exceed values for mechanical pre-stress, requiring dynamic solutions; surface temperature enhancements at changes in current direction; possibility of electrical breakdown at connection of liner cassette insulator to bank insulation; need for magnetic inhibition of breakdown (MIB) between liner surface and insulator; Liner Physics - angle needed to maintain current contact between liner and glide-plane/electrode without jetting or buckling; nonlinear magnetic diffusion into liner and associated melting; Diagnostics - X-radiography through cassette insulator and outer conductor without shrapnel damage to film.

\footnotetext{
*Work supported by the US Department of Energy under contract W-7405-ENG-36 to Los Alamos National Laboratory, Los Alamos, NM, operated by the University of California . As an institution, LANL does not endorse the viewpoint of a publication or guarantee its technical correctness.

1. Bechtel-Nevada, Las Vegas; 2. Bechtel-Nevada, Los Alamos; 3. SAIC, Albuquerque; 4. NumerEx.
}

\section{INTRODUCTION}

Pulsed power involves the compression of energy in time and space usually to achieve conditions not accessible by steady delivery of power. Typical applications of pulsed power include the creation of high energy-density states of matter for studies of material behavior, equations-of-state, and dynamics. Electromagnetically-driven implosion of cylindrical shells, aka, liners, represents a technique for creating conditions of very high pressure ( $>$ Mbar) and high speeds $(>\mathrm{km} / \mathrm{s})$. One form of this technique interacts axial current flow in a cylindrical metal liner, with its azimuthal magnetic field to create a radial force that implodes the liner. Subsequent impact of the liner with interior target material can drive strong shocks. The present paper discusses pulsed power and experimental arrangements for operating such imploding liners at peak currents of $16 \mathrm{MA}$ in order to study shock dynamics.

\section{BASIC DESIGN}

For these experiments, the Shiva Star capacitor bank [1] at the Air Force Research Laboratory, Kirtland AFB, $\mathrm{NM}$, provides the source of pulsed power at megajoule, multi-megampere levels. It consists of six arms of capacitors, connected to the central load region by flat, aluminum, transmission plates separated by Mylar and polyethylene. Shiva Star operation consists of charging the bank capacitance $\quad(\mathrm{C}=1330 \mu \mathrm{F})$ to the desired initial voltage, and triggering a plurality of multichannel, railgap switches to discharge the accumulated electrical energy. Parallel-plate transmission lines connect near the centerline of the system through the coaxial arrangement of the liner and return conductor.

In the present work, with solid-density liners, nominal conditions are: $\mathrm{V}_{\mathrm{o}}=80 \mathrm{kV}, \mathrm{J}_{\text {peak }}=16 \mathrm{MA}, \mathrm{t}_{\text {rise }}$ $=8 \mu \mathrm{s}$. Previous work on Shiva Star at comparable conditions had successfully imploded solid-density liners with initial diameters and lengths of $8-10 \mathrm{~cm}$ and $4-5 \mathrm{~cm}$, respectively [2]. Two important differences in the present design include use of 1100-, instead of 6061- 
series, aluminum for the liners, and solid-dielectric insulation, instead of vacuum in the power flow channel adjacent to the dynamic load. These changes allowed connection of the present design to future program requirements.

To achieve the desired conditions for the shock wave in the target, while remaining within previous experience, the following initial dimensions were selected for the liner: outer diameter, length and thickness of $10 \mathrm{~cm}, 4 \mathrm{~cm}$ and $1 \mathrm{~mm}$, respectively. Figure 1 displays the mechanical arrangement of the liner implosion system installed as a pre-assembled unit, the liner cassette, on Shiva Star. On the centerline, a target cartridge is inserted, consisting of a tin cylinder, $4 \mathrm{~cm}$ in diameter, within which is embedded a cylinder of acrylic, $3 \mathrm{~cm}$ in diameter (as sketched in Fig. 2). To examine both symmetrical and asymmetrical shock motion, the axis of the acrylic cylinder is either coincident with that of the tin or displaced $4 \mathrm{~mm}$ along a diameter. (In both types of test, the outer surface of the tin is coaxial with the aluminum liner.) The glideplane/electrodes along which the liner implodes each have a $10^{\circ}$ angle, so the liner impacts the target along a length of about $3 \mathrm{~cm}$. For an initial bank voltage of 82 $\mathrm{kV}$, design calculations indicate a current peak of about $16 \mathrm{MA}$ and an impact speed on the target surface of approximately $6.25 \mathrm{~km} / \mathrm{s}$. This results in a pressure in the tin of about one megabar.

The goals of the experiment included:

1) achievement of excellent axial uniformity and azimuthal symmetry of liner implosion onto target at high impact pressures.; and 2) generation of sufficiently precise data on shock motion and shape to allow detailed comparison with theoretical and numerical predictions. Diagnostics comprised: bank current measured by Faraday rotation and Rogowski coils; liner shape in rzplane imaged on film by side-on $X$-radiography; time and azimuthal symmetry of impact of liner on target recorded by array of $B$-dot coils; motion and shape of shock in acrylic indicated by optical shadowgraphy of disruption of transmission through acrylic; motion and shape of shock in acrylic, density variation behind the shock, and motion and shape of the acrylic-tin interface imaged on CCD by axial X-radiography, (see Fig.3).

\section{PULSED POWER CONCERNS}

Operation at multi-megampere currents in the microsecond regime presents several design challenges [3]. These include connection of conductors and transmission plates at high magnetic pressure, current density concentrations, and attachment of the liner to the glide-plane/electrodes at near-megagauss levels. Furthermore, joints in electrical insulation must function at tens of kilovolts for an apparatus installed in an existing pulsed power system. Additional pulsed power design issues include surface temperature enhancements

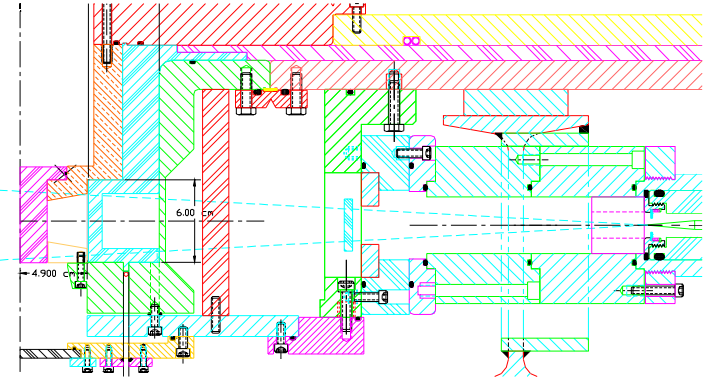

Figure 1. Mechanical drawing of the experimental arrangement showing details of the connections of conductors and the overlap joint of the polyethylene insulator disc between the Shiva Star transmission plates and the polyethylene insulator of the coaxial load assembly (the "liner cassette"). The aluminum liner overlaps the outer edges of the copper glideplane/electrodes that angle inward to the target "cartridge" cylinder. Also shown are one of the two sideon X-radiography sources and one of the array of six Bdot probes monitoring the magnetic field outside the liner surface. The latter indicate the time of liner impact.

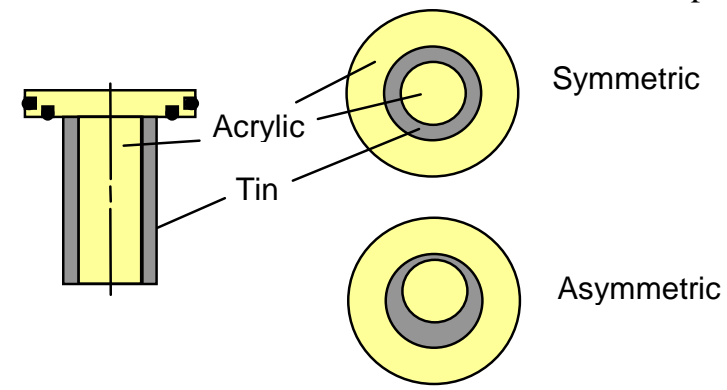

Figure 2. Sketches of target cartridge in symmetric and asymmetric forms. Note that outer surface of the tin is coaxial with liner implosion in both cases.

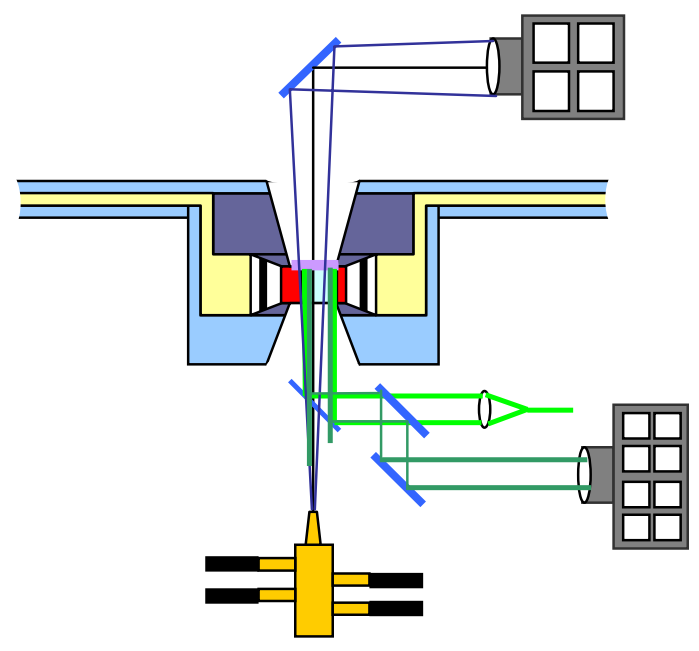

Figure 3. Schematic arrangement of the experiment, showing the axial $\mathrm{X}$-radiography and double-pass optical shadowgraphy through the target region. 
at changes in current direction, and the need for magnetic inhibition of breakdown (MIB) in the region between the liner and insulator surfaces.

Design concerns extend to the physics of the liner implosion in regard to the angle needed to maintain current contact between the liner and glideplane/electrodes without jetting or buckling, and to nonlinear magnetic diffusion into the liner and associated melting. Also, while the complement of diagnostics has been used on similar experiments, side-on X-radiography in the present arrangement operates through the cassette insulator and outer conductor, so protection of the film pack from shrapnel occurs in a new environment. At a more mundane level, use of 1100-series aluminum for the liner exacerbates the traditional problem of closely registering the centers, planes and altitudes of the upper and lower connections of a high energy, low inductance capacitor bank and the central load.

\section{A. Current Joints}

At 16 MA, the peak magnetic field levels for the current joints of the liner cassette increase from $17.4 \mathrm{~T}$ at the connection of the return conductor and the lower plate of Shiva Star (see Fig. 1), to $43 \mathrm{~T}$ at the connection of the larger, copper electrode to the upper plate of Shiva Star. Fields exceed $64 \mathrm{~T}$ at the smaller, copper electrode connection and at the liner attachment to the glideplane/electrodes. The relatively modest value of magnetic field at the return conductor connection encouraged the use of two concentric sets of bolts in a clamp ring across the junction with Shiva Star. This clamp ring enclosed a copper gasket into which triangular ridges on the aluminum conductors bite as the bolts are engaged. The dimensions of the ridges, gasket and clamp ring are chosen to permit $0.5 \mathrm{~mm}(\sim 20$ mils $)$ of inconsistency between the positions of the upper and lower plates of Shiva Star without affecting the registration of the glide-plane/electrodes holding the liner.

At the higher field connections of the aluminum conductors to the copper glide-plane/electrodes, steel bolts (at up to $3000 \mathrm{lbf} / \mathrm{bolt}$ ) are used with Helicoil inserts in the copper. This pre-stresses the contacts to limit of elastic strength $(\sim 45 \mathrm{kpsi})$, which is still less than the peak magnetic pressure $(108 \mathrm{kpsi})$ at these contacts. At each contact, the aluminum portion overlaps the copper in a manner [3] that permits the magnetic pressure to provide dynamic loading of the contact region during the rise of the current.

\section{B. Liner Connection to Glide-plane/Electrodes}

For the still higher magnetic fields at the initial, outer radius of the liner, dynamic compression becomes the only technique for maintaining current connection. In the present design, the initial contact between the aluminum liner and the copper electrodes occurs by thermally established, mechanical interference (aka, "thermal interference"). Detailed machining, at the tenth-mil level, permits successful operation [4].

\section{Temperature Enhancements}

At locations along the current path, especially near joints and the liner connections to electrodes, current concentrations should be avoided to prevent enhancement of skin-current heating, which is already a great difficulty at high magnetic fields. Thus, the present design includes radii-of-curvature much greater than the flux skin-depth at concave corners of the conducting path in the liner cassette.

\section{Insulator Connection to Shiva Star}

Installation of the liner cassette in Shiva Star requires a mechanical joint at the respective polyethylene insulators. General experience with such joints underscores the need to eliminate any low density (e.g., air) gap between the mating surfaces. In the present design, a layer of Vi-Sil (Visilox) ${ }^{\mathrm{TM}}$, a room-temperature vulcanized (RTV) material, fills potential voids both along the $5 \mathrm{~cm}$ of insulator overlap and around the $60 \mathrm{~cm}$ circumference of the insulators. A total force of $2000 \mathrm{lbf}$ flattens and compresses the overlap region.

\section{E. Insulation Adjacent to Liner}

In previous work on Shiva Star, the solid-dielectric insulation between the transmission plates ends at a considerable distance $(>10 \mathrm{~cm}$ ) from the load region. Indeed, a major success in the development of high power, plasma liner implosions for soft X-ray production in the Air Force Shiva program derived from separating the vacuum-plastic interface of the solid insulator from the UV-radiating, plasma liner. With solid-density liners, however, such separation is not required, (even though it has persisted for convenience of experimental design). The present arrangement places the final insulator surface as close as possible to the initial outer surface of the liner. This maximizes the opportunity for magnetic inhibition of breakdown (MIB) in the lower density material between the liner and the insulator [3].

\section{SUMMARY OF RESULTS}

For all four shots of the experimental series, the pulsed power and experimental apparatus operated in accordance with design expectations. Fabrication of the liner cassette, including the thermal interference connections of the liner to the glide-plane/electrodes, was accomplished successfully. The assembly procedure proved satisfactory in achieving installation of the liner cassette in Shiva Star. The copper-gasket connection provided adequate margin for registering the electrodes and the relatively delicate 1100 -series aluminum liner, and the RTV material flowed and filled in discrepancies at the insulator joint. All the current joints and connections worked in delivering the bank current to the liner. In accord with calculations, the current rose sinusoidally in $8 \mu$ s to values between 15.7 and $16 \mathrm{MA}$, and the liner achieved a radial speed of $6.25 \mathrm{~km} / \mathrm{s}$ at the time of impact on the target cartridge. Impact of the liner on the tin surface provided high-frequency signals to the array of six magnetic probes surrounding the implosion 
indicating an azimuthal symmetry of arrival of $10 \mathrm{~ns}$ after $13.5 \mu$ s of travel.

Side-on X-radiography successfully captured images of the liner on photographic film that survived the shrapnel environment of the implosion event. The liner appeared as a smooth, right-circular cylinder, except for small $(<\mathrm{mm})$ triangular regions leading and trailing the liner at its contacts with the glide-planes, (see Fig. 4). $\mathrm{MACH} 2$ numerical simulations of the liner implosion display similar behavior and features, (see Fig. 5). The side-on radiography also imaged the liner after impact with the target cartridge, (see Fig. 6). In addition to the larger bulge associated with the earlier triangular features on the glide-plane, the radiograph displays fine-scale structures extending radially at many points along the outer surface of the liner after impact. The larger bulge is also seen in the MACH2 result displayed in Fig. 7.

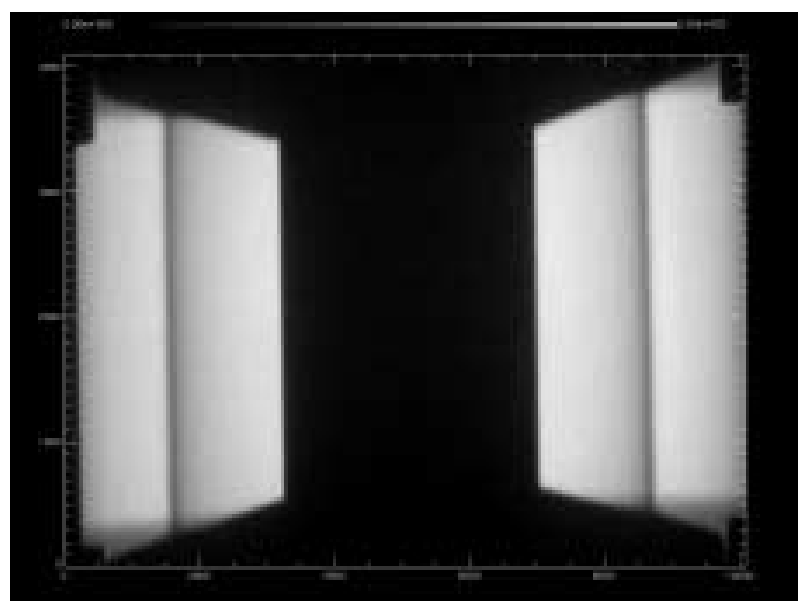

Figure 4. Side-on X-radiograph of liner implosion on first shot of NTLX series on Shiva Star. The liner is straight and slightly more than $1 \mathrm{~mm}$ thick, except at small triangular regions at the glide-planes. The dark region in the center is the target cartridge.

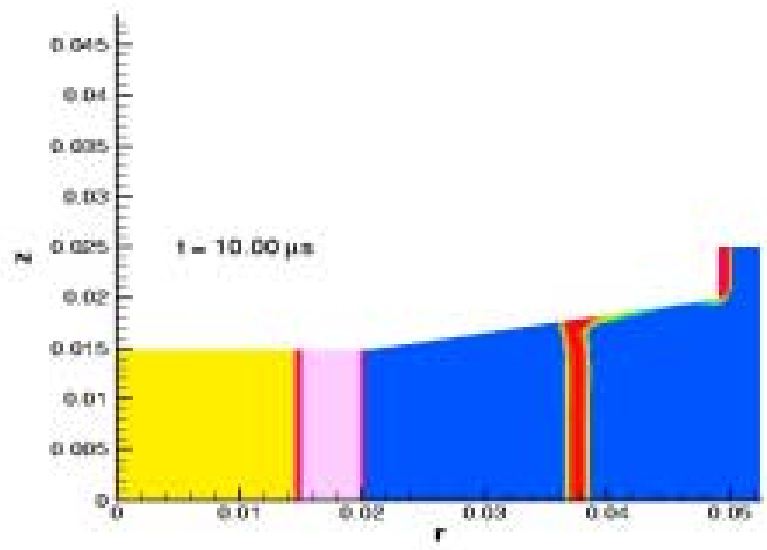

Figure 5. MACH2 code simulation of liner implosion of Fig.4 indicating liner contact along glide-plane. Note thinning of material on glide-plane surface after corner.

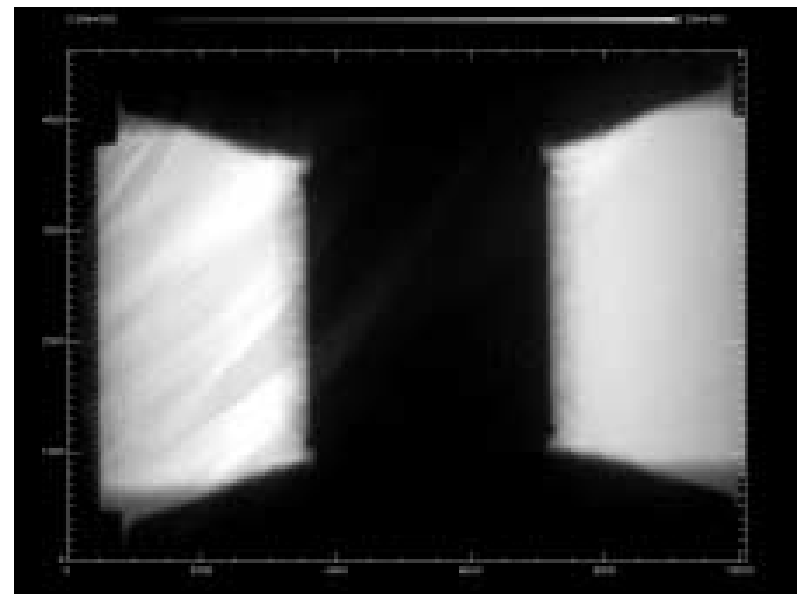

Figure 6. Side-on radiograph after impact of liner (of Fig.5) on target. Note larger bulges near glide-planes.

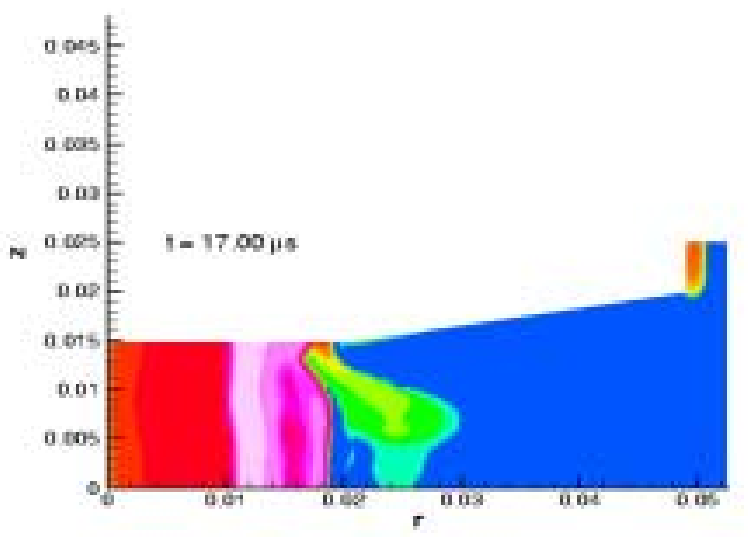

Figure 7. Close-up of MACH2 code simulation for liner implosion of Figs. 4 and 6 showing details of impact on target of triangular features of Fig. 5.

Companion papers elsewhere in these proceedings provide additional results and discussions of these experiments.

\section{REFERENCES}

[1] R.E. Reinovsky, et al, "Shiva Star Inductive Pulse Compression System," in Proc. of 4th IEEE Intern.

Pulsed Power Conf., Albuquerque, NM, T.H. Martin and M.F. Rose, eds., IEEE Cat. No. 83CH1908-3 (1983). P. 196.

[2] J.H. Degnan, et al, "Compression of Plasma to Megabar Range Using an Imploding Liner,” Phys. Rev. Letters, 82, 13, 2681 (1999).

[3] P.J. Turchi, "Problems and Prospects for Microsecond Pulsed Power Above Ten Megamperes," IEEE Trans. on Plasma Science, Vol. 28, No. 5, Oct 2000. Pp. $1414-1421$.

[4] W. Anderson, et al, “A Proposed Atlas Liner Design Fabricated for Hydrodynamic Experiments on Shiva Star," these proceedings. 